\title{
Localization of thermal sensation: An illusion and synthetic heat
}

\author{
BARRY G. GREEN \\ John B. Pierce Foundation Laboratory and Yale University, New Haven, Connecticut 06519
}

\begin{abstract}
Touching three thermal stimulators with the first, second, and third fingers of the hand revealed the following phenomena: (1) When the outer two stimulators were warm (or cold) and the center stimulator thermally neutral, warmth (or cold) was felt at all three fingers (referral). (2) When all three stimulators were warm (or cold), the magnitude of warmth (or cold) felt at the middle finger was greater than the sensation felt when the center stimulator alone was touched (enhancement). (3) When the thermal qualities of the center and outer stimulators differed, the sensation at the middle finger often took on the quality of the sensation produced at the outer fingers (domination). (4) Synthetic heat was sometimes experienced when the outer stimulators were warmed and the center stimulator cooled. The results raise interesting possibilities concerning the probable role that tactile stimulation plays in thermal localization.
\end{abstract}

Localization of thermal sensation seems from experience to be unerring and absolute. We know with certainty which finger touches a hot stove and which side of the face the sun warms. But contrary to these experiences are the results of numerous experiments on thermal localization and discrimination. With few exceptions (Békésy, 1962), experiments show that localization of thermal stimuli is abysmally poor compared to localization of tactile stimuli (Cain, 1973; Dimmick, 1915; Pritchard, 1931; Stone, 1937; Taus, Stevens, \& Marks, 1975).

Why this discrepancy between experience and experiment? The answer seems to be that relatively few pure thermal stimuli (i.e., from radiant sources) are encountered in daily life, and when they are the spatial discriminations possible are relatively gross: -Knowing which side of the face is warmed by the sun seems an unimpressive achievement when the error of localization for pressure on the lip is as small as $1 \mathrm{~mm}$ (Weinstein, 1968). Even grosser localizations (e.g., front-back discriminations) sometimes fail when radiant heat alone is the stimulus (Cain, 1973).

It is when thermal stimuli occur in conjunction with stimuli that appeal to other modalitiesparticularly touch-that localization improves. In such cases, it may be misleading to speak of thermal localization per se: Ordinarily, we touch an object before we perceive its thermal qualities, and to the extent the object touched is localized, so too is the accompanying thermal sensation. After finding

This work was supported in part by a training grant from the National Institutes of Health, No. ES-00123-08, while the author was a postdoctoral fellow at Yale University. Reprint requests may be sent to: Barry G. Green, John B. Pierce Foundation Laboratory, 290 Congress Avenue, New Haven, Connecticut 06519. localization of radiant heat stimuli much inferior to localization of tactile stimuli, Pritchard (1931) concluded that "It is only when the ... stimulus ... involves mechanical deformation of the skin that accurate localization is possible."

The present study supports Pritchard's contention by demonstrating the mislocalization of thermal sensations when the spatial pattern of thermal stimulation is inconsistent with the spatial pattern of tactile stimulation. Experiment 1 investigates the effect of touching warm (or cold) surfaces with the first and third fingers while simultaneously touching a thermally neutral surface with the second finger. The result is a sensation of warmth (or cold) at the tip of each finger. Two other experiments investigate other aspects of the "illusion," including conditions that sometimes induce sensations of "heat."

\section{EXPERIMENT 1}

\section{Method}

Subjects. Ten women and one man, between the ages of 18 and 24 , served as paid subjects. All but one were inexperienced in the method of magnitude estimation; all were naive to the purpose of the experiment.

Apparatus. Thermal stimuli were generated by three Peltier thermoelectric modules (Cambion Electronics, No. 801-3959-01) measuring $21.5 \mathrm{~mm}$ square. The modules were mounted on hollow copper cylinders, 1 in. in diameter and $30 \mathrm{in}$. high, which acted as heat sinks. Water from a constant temperature bath $\left(30^{\circ} \mathrm{C}\right)$ circulated through the cylinders. The cylinders stood on an aluminum base and were spaced $\mathbf{4 0} \mathrm{mm}$ apart, center to center. The temperatures of the modules were controlled independently via 10-A, continuously variable power supplies equipped with polarity switches. A thermocouple glued to the surface of each module enabled display of the temperature of the stimulators on a Honeywell two-channel recorder. A switch allowed selection of which two module temperatures were displayed. Temperatures were set with an accuracy of about $\pm 0.5^{\circ} \mathrm{C}$. 
A water bottle filled with $30^{\circ} \mathrm{C}$ water served as a preadapting stimulus.

Procedure. The subjects were seated at a table with the stimulators and water bottle placed before them. They were instructed to place their preferred hands on the water bottle to begin preadaptation. Written instructions were provided describing the basic procedure for magnitude estimation, followed by verbal instructions to touch the tips of the first three fingers of the preferred hand to the three stimulators simultaneously, and to pay strict attention only to sensations perceived at the middle finger (center stimulator). Subjects were reminded of this requirement early in the session. The session began when the subject felt the preadapting stimulus as thermally neutral.

Subjects touched the stimulators for $3 \mathrm{sec}$ per trial, and returned the hand to the preadapting stimulus between trials. Magnitude judgments reflected the maximum sensation perceived during a trial.

The experiment included two conditions: (1) a control condition, in which the temperature of the center stimulator was varied and the temperature of the outer stimulators was constant at $30^{\circ} \mathrm{C}$, and (2) the experimental condition, in which the temperature of the center stimulator was constant at $30^{\circ} \mathrm{C}$ and the temperature of the outer stimulators was varied. Both warm $\left(33^{\circ}, 36^{\circ}, 39^{\circ}, 42^{\circ}\right.$, $\left.44^{\circ} \mathrm{C}\right)$ and $\operatorname{cool}\left(9^{\circ}, 13^{\circ}, 17^{\circ}, 21^{\circ}, 25^{\circ} \mathrm{C}\right)$ stimuli were tested.

Approximately half the subjects served first in the control condition; the remaining subjects served first in the experimental condition. Stimulator temperatures were presented randomly within a condition until two magnitude estimates were obtained at each temperature. Two estimates were then obtained at each temperature in the remaining condition. After a brief rest, the sequence was repeated, yielding four magnitude estimates per temperature in each condition. Subjects served in 21-h sessions on separate days, a single session including only warm or only cool stimuli.

\section{Results}

Geometric means of magnitude estimates of perceived warmth and cold are plotted in Figure 1 (A and $B$ ) as a function of stimulator temperature. Each point is based on 44 observations. The filled circles in Figure $1 \mathrm{~A}$ represent magnitude estimates of the perceived cold at the tip of the middle finger when the temperature of the center stimulator alone varied (control condition). The empty circles represent magnitude estimates of the perceived cold at the tip of the middle finger when the temperature of the outer stimulators alone varied (experimental condition).

In all cases, the perceived magnitude of cold at the middle finger in the experimental condition was similar to the perceived magnitude of cold at the middle finger in the control condition, this despite the absence in the experimental condition of a thermal stimulus at the middle finger. Figure 1B shows similar results for warmth: The perceived magnitude of warmth in the experimental condition equaled or exceeded the perceived magnitude of warmth at the middle finger in the control condition.

The data in Figure 1 suggest that the thermal sensation localized at the middle finger in the experimental condition is comparable to the sensation evoked by direct stimulation of the middle finger. This "referral" of sensation to the middle finger appeared so genuine that none of the 11 subjects realized that on half of the trials the middle stimulator was thermally neutral. Indeed, in both the control and experimental conditions, one is quite confident that the thermal sensations originate at the tip of each finger. This point is demonstrated further by the requirement that the middle finger contact the center stimulator in order to perceive a thermal sensation at its tip: Kept just above the stimulator the middle finger retains thermal neutrality. The phenomenon of "externalization" of sensation (i.e., localization of sensation between fingers), noted by Békésy (1962), was never reported.

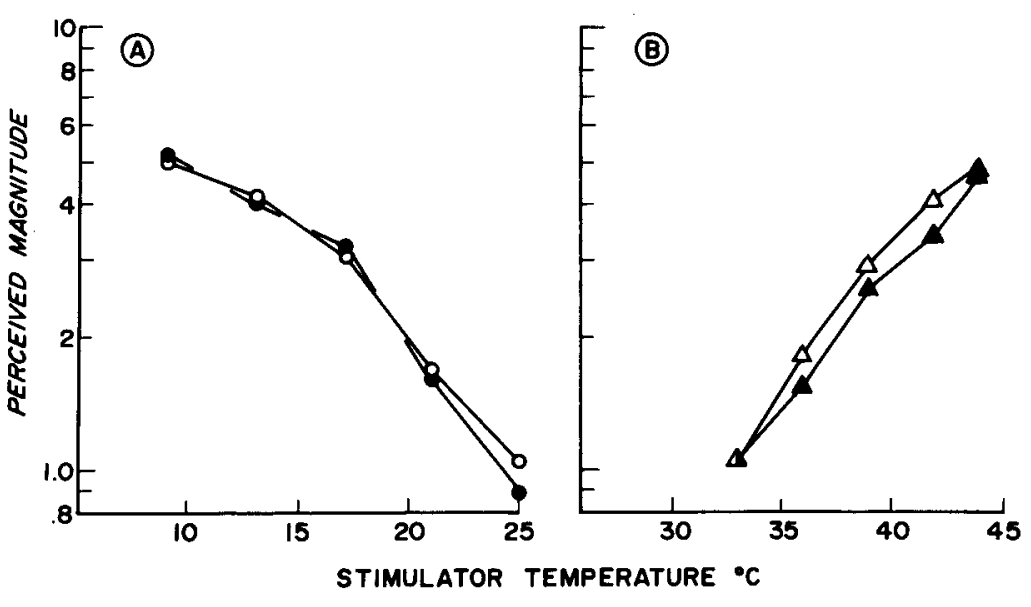

Figure 1. Part A shows the perceived magnitude of cold sensation at the middle finger as a function of stimulator temperature, with the temperature of the center stimulator alone varied (filled circles) or with the temperature of the outer stimulators varied and the center stimulator held at $30^{\circ} \mathrm{C}$ (open circles). Part B shows the perceived magnitude of warmth sensation at the middle finger as a function of stimulator temperature, with the temperature of the center stimulator alone varied (filled triangles) or with the temperature of the outer stimulators varied and the center stimulator held at $30^{\circ} \mathrm{C}$ (open triangles). 


\section{EXPERIMENT 2}

The next step was to determine what effect, if any, the referred sensation had upon the sensation evoked by an actual thermal stimulus. Is the referred sensation abolished by the presence of thermal stimulation at the site of referral, or do the referred and "veridical" sensations sum to produce a sensation magnitude greater than that produced by either alone? The present experiment sought to answer this question.

\section{Method}

Subjects. Two men and 14 women (between 18 and 26 years of age) served as paid subjects. Both of the men and 4 of the women were new to the task; the remaining 10 women had served in Experiment 1 . None had knowledge of the results of the first experiment, nor did any know the purpose of the present experiment.

Apparatus and Procedure. The apparatus and instructions were the same as in Experiment 1.

The procedure included three conditions: (1) a condition identical to the control condition in Experiment 1; (2) a condition in which the temperature of the center stimulator was varied while the outer stimulators were kept at a moderate temperature $\left(36^{\circ}\right.$ or $21^{\circ} \mathrm{C}$ ); and (3) a condition like (2) except that the outer stimuli were held at a more extreme temperature $\left(44^{\circ}\right.$ or $\left.9^{\circ} \mathrm{C}\right)$. The conditions were run in random order across subjects, and the experiment was again divided into two 1 -h sessions, one for warm stimuli and one for cold.

Temperatures presented to the middle finger were the same as in Experiment $1\left(33^{\circ}, 36^{\circ}, 39^{\circ}, 42^{\circ}\right.$, and $44^{\circ} \mathrm{C}$ for warm; $9^{\circ}$, $13^{\circ}, 17^{\circ}, 21^{\circ}$, and $25^{\circ} \mathrm{C}$ for cold). Two observations were recorded in each condition at each temperature for a total across all subjects of 32 observations per point.

\section{Results}

The presence of a warm or cool stimulus at the middle finger failed to abolish the referred thermal sensation. Instead, Figure 2 shows that touching a warm or cool surface with the first and third fingers enhances the perceived warmth or cold of a surface touched by the second finger. As the converging functions imply, this enhancement becomes proportionally less as the temperature of the center stimulator becomes more extreme. ${ }^{1}$ The flatter slopes of the curves for the $9^{\circ} \mathrm{C}$ and $44^{\circ} \mathrm{C}$ outer temperatures also mean that very cold and very warm outer stimuli tend to reduce the effectiveness of stimulation at the middle finger: The referred sensation produced by extreme temperatures overwhelms the veridical sensation produced by mild temperatures.

\section{EXPERIMENT 3}

The results of Experiments 1 and 2 demonstrate that sensations of warmth and cold can be referred across fingertips when the site of referral is either thermally neutral or has the same thermal quality (warmth or cold) as the outer sensations. This experiment asks: If the thermal qualities differ between middle and outer fingers, will referral still occur? If referral occurs, which quality will dominate, that of the veridical sensation or that of the referred sensation?

\section{Method}

Subjects. Fourteen women and one man (ages 18-26) served as paid subjects. All had served in Experiment 2, and all remained naive to the results and purpose of the experiments.

Apparatus and Procedure. The apparatus was the same as in Experiment 2.

Instructions to the subjects were also the same as before, except that, in addition to assigning a number corresponding to the perceived magnitude of sensation, subjects were asked to choose a label corresponding to the perceived quality of sensation. A list of six labels (very cold, cold, cool, warm, very warm, and hot) was provided. The subjects were urged to judge sensation magnitude independent of quality (i.e., to avoid limiting magnitude judgments to six categories), and to report only the quality localized at the middle finger if the qualities between the middle and outer fingers differed. Additional comments about the quality of sensations were noted when reported.
(A)

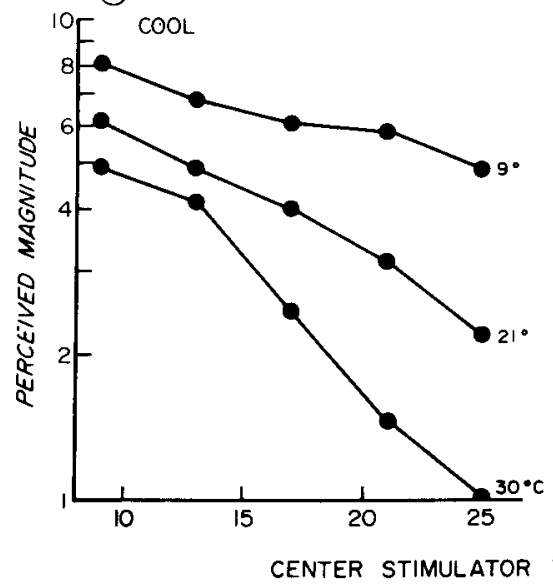

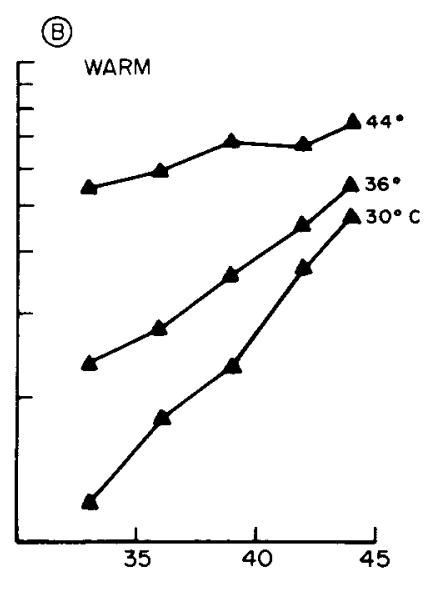

Figure 2. Perceived magnitude of cold (A) or warmth (B) at the middle finger as a function of center stimulator temperature. The parameter is the temperature of the outer stimulators. 
The experiment included three conditions: (1) a control condition identical to those of Experiments 1 and 2; (2) a "warm" condition, in which the outer stimulators were set at $44^{\circ} \mathrm{C}$ and the temperature of the center stimulator varied; and (3) a "cold" condition, in which the outer stimulators were set at $9^{\circ} \mathrm{C}$ and the temperature of the center stimulator was again varied. In each condition, the center stimulator was adjusted randomly over the following temperatures: $9^{\circ}, 17^{\circ}, 21^{\circ}, 25^{\circ}, 33^{\circ}, 39^{\circ}$, and $44^{\circ} \mathrm{C}$. Thus in Conditions 2 and 3 , the outer stimulators and the center stimulator should have produced sensations of opposing thermal quality on approximately half of the trials.

The order in which the conditions were presented was randomized across subjects, and all three conditions were completed in a 1 -h session. Two trials were run at each center stimulator temperature in each condition, for a total over all subjects of 32 judgments of perceived magnitude and sensation quality at each temperature.

\section{Results}

Figure 3 displays the results of the magnitude estimation portion of the experiment. The control condition (filled circles) yielded results consistent with Experiments 1 and 2, and in addition allowed comparison of the perceived magnitude of thermal sensations on both sides of thermal neutrality (warm and cold stimuli were presented in the same session). Note that a $44^{\circ} \mathrm{C}$ center stimulator produced nearly the same perceived magnitude as that produced by a $9^{\circ} \mathrm{C}$ center stimulator. Thus, the coldest cold and the warmest warm had equivalent sensation magnitudes.

In cases where outer and center stimulator temperatures were similar (both warm or both cold, Figure 3, open triangles above $30^{\circ} \mathrm{C}$, open circles below $30^{\circ} \mathrm{C}$ ), the results of Experiment 2 were replicated. Warm outer stimuli enhanced judgments of perceived warmth when the center stimulator was warm, and the effect of the outer stimuli became proportionally smaller at more extreme temperatures. The same was true for cold. Also interesting was the result of pairing $9^{\circ} \mathrm{C}$ outer stimulators with a $9^{\circ} \mathrm{C}$ center stimulator, and $44^{\circ} \mathrm{C}$ outer stimulators with a $44^{\circ} \mathrm{C}$ center stimulator: the two pairings gave rise to similar sensation magnitudes. This is the expected result if $9^{\circ} \mathrm{C}$ and $44^{\circ} \mathrm{C}$ produce equally intense sensations (which they do), and if warmth and cold show similar amounts of referral, as the results of Experiment 1 imply they do.

Conditions involving disparate thermal qualities (e.g., warm outer stimulators and cold center stimulator), gave somewhat different results for warmth and cold. The $44^{\circ} \mathrm{C}$ outer stimuli enhanced the perceived magnitude of the sensation at the middle finger when that finger touched a cool or cold stimulator (open triangles below $30^{\circ} \mathrm{C}$ in Figure 3). At $21^{\circ}$ and $25^{\circ} \mathrm{C}$, warm outer temperatures seem even more effective than cool outer temperatures in enhancing sensation at the middle finger. These surprising results imply that even when adjacent thermal sensations differ in quality, their perceived magnitudes may interact.

The interaction between warmth and cold appears, however, to be asymmetric. That is, cold appears to refer less well to the site of a warm stimulus than does warm refer to the site of a cold stimulus. The open circles above $30^{\circ} \mathrm{C}$ in Figure 3 show the result of combining a cold referred sensation (produced by $9^{\circ} \mathrm{C}$ outer temperatures) with a warm veridical sensation: All points fall below the function resulting from the combination of a warm referred sensation with a warm veridical sensation. The $9^{\circ} \mathrm{C}$ outer stimulators leave the sensation magnitude produced by a $44^{\circ} \mathrm{C}$ center stimulator almost unaltered. We may conclude that the perceived magnitude of a warmth sensation is enhanced less by surrounding sensations of cold than by surrounding sensations of warmth.

The results of the labeling task are shown in Fig-

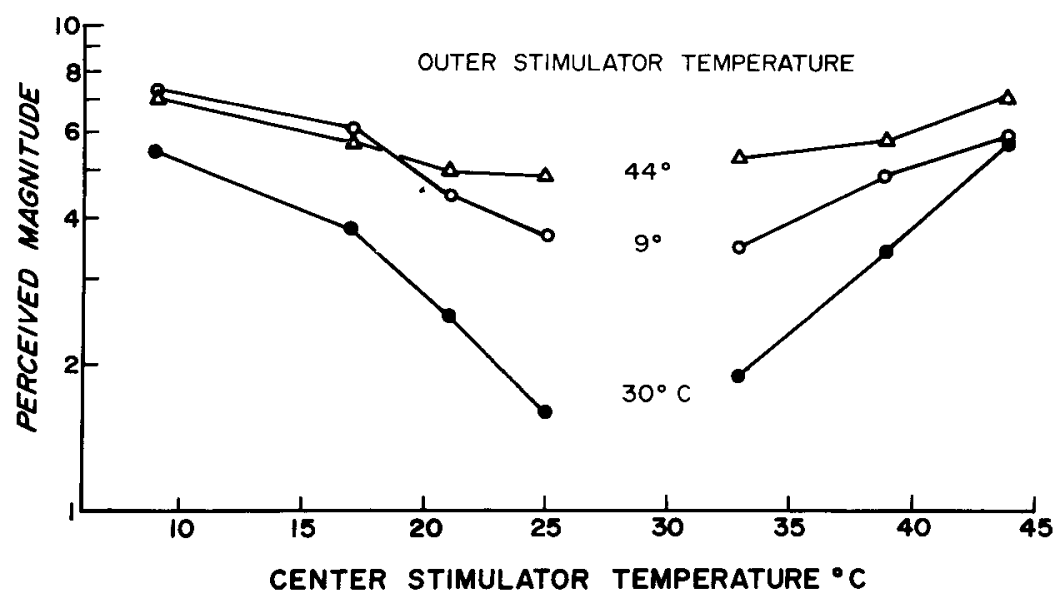

Figure 3. Perceived magnitude of the thermal sensation at the middle finger as a function of center stimulator temperature. The parameter is the temperature of the outer stimulators. The open circles on the right and the triangles on the left represent conditions in which the quality of the thermal sensations aroused by the center and outer stimulators differed. 
ure 4, which graphs the percent judgments of warmth (A) and the percent judgments of cold (B) as a function of the temperature of the center stimulator. The square symbols (both filled and open) show the judgments of quality in the control condition: Not surprisingly, percent judgments of warmth (A) increase monotonically with rise in temperature, and percent judgments of cold (B) increase monotonically as temperature falls. More surprising are the results for disparate thermal qualities. The closed circles (A) show the percent warmth judgments in the presence of an outer temperature of $44^{\circ} \mathrm{C}$. The warm outer temperature shifted the curve for judgments of warmth to the left. That is, even when the center stimulator was cooled to $9^{\circ} \mathrm{C}$, the resulting sensation was given one of the three "warm" labels on $30 \%$ of the trials. The opposite (but commensurate) trend was obtained with $9{ }^{\circ} \mathrm{C}$ outer temperatures (Figure $4 \mathrm{~B}$, open circles): When the center stimulator was warmed to $44^{\circ} \mathrm{C}$, the sensation was given one of the three "cool" labels on $37 \%$ of the trials. Hence the thermal quality aroused by the outer stimuli sometimes "dominated" the opposing thermal quality localized at the center stimulus.

"Domination," however, fails to describe the changes in thermal quality that occur in some conditions in which warm outer temperatures change the perception of a cool center stimulus. Under these conditions, the quality of "heat" was sometimes produced. Heat has been characterized as a synthesis of warm and cool that is qualitatively different from either (Alrutz, 1898). Typically produced by presenting a grill of alternating warm and cool elements to the skin (Cutolo, 1918), heat is thought to be similar to the sensation produced when skin temperature is raised to just below the threshold for pain. Alrutz (1898) believed it was caused by the simultaneous activation of cold receptors at high temperatures (paradoxical cold) along with adequate stimulation of warm receptors at the same site on the skin. In the present situation, it appears that thermal localization is so poor that warm and cool stimuli presented to separate fingers provide afferent signals that resemble the signals provoked by very warm temperatures at a single finger.

Figure 5A provides a better picture of the frequency of occurrence of sensations of heat, showing the percent of warm responses labeled either "hot" or "very warm" (broken line). Of particular note is the nonmonotonicity of the function-a secondary peak occurs at $21^{\circ} \mathrm{C}$, where $33 \%$ of the warm responses were "hot" or "very warm." Thus, as temperature decreased from $44^{\circ} \mathrm{C}$, the frequency of judgments of extreme warmth first declined, and then rebounded at moderately cool temperatures before declining again at the coldest temperatures.
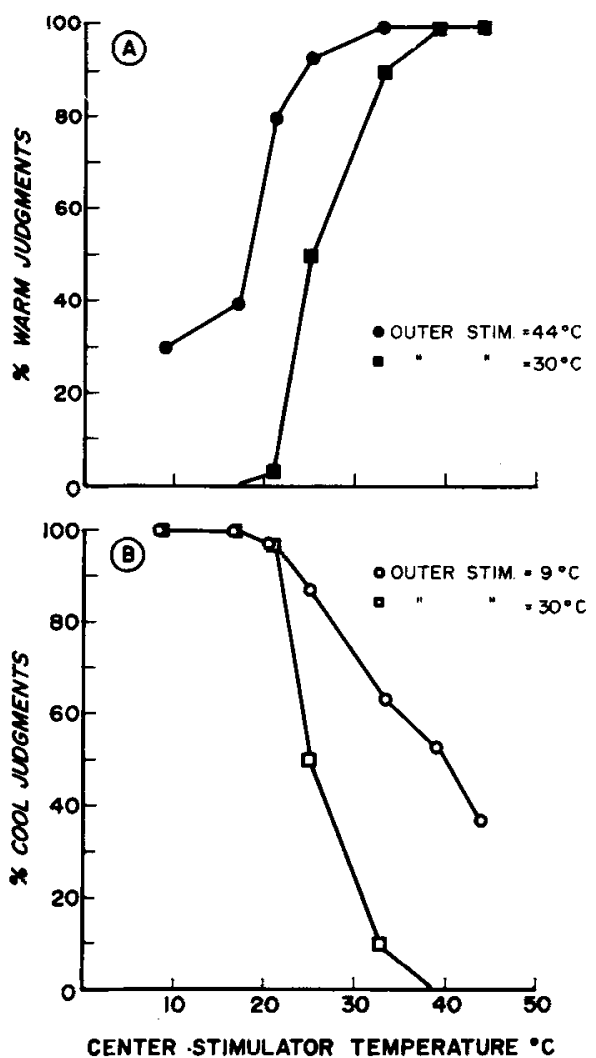

Figure 4. The percent warm judgments (A) and percent cool judgments (B) as a function of center stimulator temperature. The parameter is the temperature of the outer stimulators.

The peak at $21^{\circ} \mathrm{C}$ may reflect the optimal condition (in the confines of the experiment) for production of synthetic heat.

A similar graph showing percent of "cold" and "very cold" judgments (Figure 5B) turns up the same kind of effect for cold outer temperatures in combination with warm center temperatures. Judgments of "cold" and "very cold" first decline with increasing temperatures, then rise in frequency at $39^{\circ} \mathrm{C}$.

Should this puzzling peak in "cold" and "very cold" judgments be considered an instance of "synthetic cold'? Present evidence argues against such a phenomenon. Casual observation reveals that sensations of cold produced under such conditions contain none of the novel qualities (e.g., burning or prickling) that characterize a synthetic quality. The sensation of warmth instead seems simply to be overwhelmed by a sensation of cold. The similarity between the functions for qualitative judgments of extreme warmth and extreme cold in Figure 5 are nevertheless very striking; those data alone provide no hint as to why spatially alternated warm and cool stimuli produce synthetic heat and not synthetic cold. 

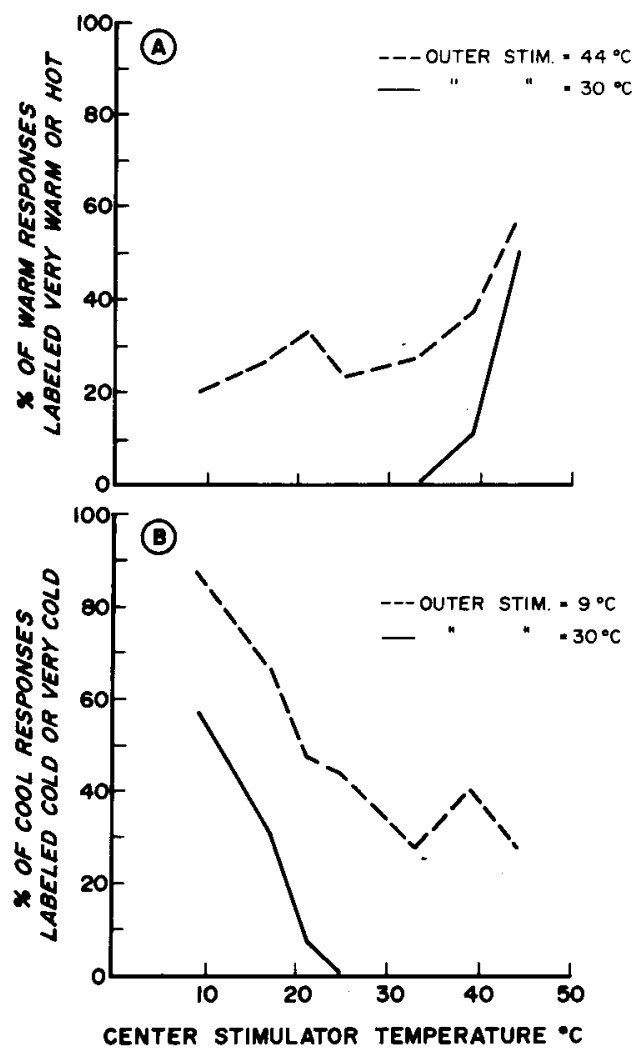

Figure 5. The percent of warm responses labeled very warm or hot $(A)$ and the percent of cool responses labeled cold or very cold $(B)$, as a function of center stimulator temperature. The dashed lines show what results when the outer stimulators are heid at $44^{\circ} \mathrm{C}$ (A) or $9^{\circ} \mathrm{C}$ (B). The solid lines were obtained with the outer stimulators held at $30^{\circ} \mathrm{C}$.

\section{DISCUSSION}

Experiment 1 exemplified the inaccuracy of thermal localization. Demonstration of the referral of sensation to a locus devoid of thermal quality, but having similar tactile quality, supports the idea that touch guides localization of thermal sensation (Pritchard, 1931). When the middle finger is held just above the stimulator, no thermal sensation is appreciated. Nor, however, does the middle finger feel warm or cool when touched by a finger of the other hand. This latter observation may mean that tactile stimulation alone is inadequate to cause referral of sensation: Perhaps referral occurs only among surfaces that produce qualitatively similar tactile sensations. This notion invites further attention.

In seeking a neurophysiological basis for the illusory sensation, one must search beyond the organization of thermal innervation in the hand. The hand was chosen for experimentation only because of its superior tactile localization and because the fingertips provide assurance of the independence of thermal stimuli. Experiments presently underway demonstrate the illusion on the forearm, and the author has experienced referral of sensation on the thigh and abdomen as well as across the second, third, and fourth fingers. The illusion seems simply to depend upon the extremely poor spatial discrimination of warmth (Cain, 1973) and cold, and upon neural interactions between the thermal and tactile modalities somewhere in the nervous system. Experiments testing for the illusion across the midline of the body can help locate the site of interaction in either the periphery or the CNS. The illusion fails to occur, for example, when the middle finger of the contralateral hand touches the center stimulator.

Experiment 2 showed that thermal sensations may be referred to sites possessing the same thermal and tactile qualities as the inducing stimuli, and that enhancement of sensation magnitude results. Warmth and cold produce similar amounts of enhancement; hence, it may be inferred that sensations of warmth and cold produced by contacting a surface rely on similar mechanisms for localization.

Experiment 3 yielded several interesting results. First, referral of sensations of warmth to the site of a cold stimulus produced greater perceived magnitudes than did referral of sensations of cold to the site of a warm stimulus. This result was unexpected given the apparent similarity in behavior of warmth and cold shown in Experiment 2. Second, when outer and center stimulators differed widely in temperature, the quality of the sensation at the middle finger was sometimes dominated by the sensation produced at the outer stimulators. An a priori equally likely outcome-namely enhancement of the veridical thermal quality as a result of simultaneous contrast-seemed never to operate in these experiments. Third, the sensation of heat was sometimes produced when a cold center stimulus was bracketed by warm outer stimuli. Instructions to subjects included no mention of sensations of heat, since such instructions might have biased their judgments. Still, subjects sometimes volunteered comments that were indicative of the perception of heat, or they described the sensation as first feeling cold, then turning very warm or hot, which is symptomatic of synthetic heat (Burnett \& Dallenbach, 1927). It seems a reasonable assumption that the plot of the percent judgments of very warm and hot (Figure 5A) reflects the frequency of perceptions of heat. But, as noted above, the same plot (Figure 5B) for cold outer stimulator temperatures and warm center stimulator temperatures challenges this assumption. Because the function in Figure 5B mirrors that of Figure $5 \mathrm{~A}$, might we be forced to conclude that synthetic cold occurs as frequently as synthetic heat? Information germane to this problem comes from reports from subjects, and from the results of the magnitude judgments in Experiment 3. 
In the disparate-temperature conditions, subjects never reported unusual sensations of cold in the way they sometimes reported unusual sensations of warmth (e.g., perceptions of cold preceded by sensations of warmth were never reported). Perhaps more important, in the disparate conditions, cold outer stimulators enhanced sensations less than did warm outer stimulators (Figure 3, open circles on the left compared to open triangles on the right). Thus, when spatially adjacent warm and cold stimuli interact, sensations of warmth seem to dominate the intensitive aspects of the sensation. Burnett and Dallenbach (1928) arrived at a similar conclusion. In developing a linear equation to describe the relationship between warmth and cold in producing heat, they found that the warmth term required weighting by a factor of three. They concluded that "the warm stimulus is more effective for changing the intensity of the sensation of heat than a change in the cold stimulus." Just why warmth should prevail over cold when the two are juxtaposed is unclear.

Much remains to be discovered about the nature of the phenomenon reported here. What role, if any, does time play in altering the quantity and quality of the referred sensation? Must the surface texture of the outer and center stimulators always be similar? What are its spatial limitations? Current experiments investigating the spatial aspects of the effect reveal that on the forearm at least, referral sometimes occurs between just $t w o$ contact thermal stimuli when the stimuli are a few centimeters apart. With only two stimulators, however, referral appears to be more severely limited spatially. This finding is being pursued, since previous attempts to define the limits of referral on the arm with three stimulators proved fruitless even when the stimulators were separated maximally from wrist to elbow. Whatever the results from further experimentation might be, the basic finding of this study-that localization of thermal stimulation is subject to modification by tactile stimulation-has important implications for any theory of localization on the skin.

\section{REFERENCES}

AlRUTZ, S. On the temperature senses: II. The sensation 'hot.' Mind, 1898, 2 ser., 7, 140-144.

BÉKÉsY, G. von. Lateral inhibition of heat sensations on the skin. Journal of A pplied Physiology, 1962, 17, 1003-1008.

Burnett, N. C., \& Dallenbach, K. M. The experience of heat. American Journal of Psychology, 1927, 38, 418-431.

Burnett, N. C., \& Dallenbach, K. M. Heat intensity. American Journal of Psychology, 1928, 40, 484.494.

CAIN, W. S. Spatial discrimination of cutaneous warmth. American Journal of Psychology, 1973, 86, 169-181.

Cutolo, F.. J J . A preliminary study of the psychology of heat. American Journal of Psychology, 1918, 29, 442-448.

Diмміск, F. L. On the localization of pure warm sensations. American Journal of Psychology, 1915, 26, 142-150.

Pritchard, E. A. B. Cutaneous tactile localization. Brain, 1931. 54. 350-371.

Stone, L. J. An experimental study of form perception in the thermal senses. Psychological Record, 1937, 1, 234-337.

Taus, R. H., Stevens. J. C., \& Marks, L. E. Spatial localization of warmth. Perception \& Psychophysics, 1975, 17. 194-196.

WeINSTEIN, S. Intensive and extensive aspects of tactile sensitivity as a function of body part, sex, and laterality. In D. R. Kenshalo (Ed.). The skin senses. Springfield, Ill: Thomas. 1968.

\section{NOTE}

1. The data are plotted in semi-log coordinates to illustrate better the convergence of functions at extremes of temperature. Convergence on semi-log coordinates means that the influence of the outer stimulators becomes proportionally less as the temperature of the center stimulator grows colder or warmer. Convergence is also obtained on linear coordinates (though less strikingly), which probably means that bracketing stimulation does not simply add a constant increment to the perceived magnitude of sensation at the middle finger.

(Received for publication May 9, 1977; accepted July 13, 1977.) 